\title{
Education and Music Culture in the Context of Web 2.0
}

\author{
https://doi.org/10.3991/ijet.v16i10.19693 \\ Vira Revenko \\ R. Glier Kyiv Municipal Academy of Music, Kyiv, Ukraine \\ viraoperalgmail.com
}

\begin{abstract}
Total digitalization of culture requires the extensive integration of Web 2.0 services and capabilities into music education. The penetration of digital technologies is not reduced to the technologies for fixing and distributing musical works or the means of their broadcasting but also dramatically transforms the process of creating musical works. Web 2.0 offers a new distributed creativity culture and provides musicians with specific opportunities to work and distribute creative products. The study is devoted to the integration of music education and the services and opportunities that Web 2.0 provides to the mass audience (free specialized and non-specialized services), its impact on the quality of music education, as well as on the most important psychological parameters of students of music universities. The study involved 150 students of R. Glier Kyiv Municipal Academy of Music (Kyiv, Ukraine); there were three groups of 50 participants: two experimental groups and a control one. In Experimental Group 1, training was partially integrated with Web 2.0 services; in Experimental Group 2, there was a more complete and structured integration. Based on the results of semester training and the objective test scores, a significant increase in the results of experimental groups was observed (an increase by 1.3 points in Group 1 and 2.84 points in Group 2 on a 5-point scale); Group 2 demonstrated a more significant improvement. The subjective assessment of the level of involvement, motivation and anxiety showed a statistically significant decrease in academic anxiety in the experimental groups (2.99 and $2.01 \mathrm{com}$ pared to 3.98 in the control group, respectively) and a statistically significant increase in motivation and involvement in the group with deep integration of Web 2.0 services (Experimental Group 2). The hypothesis of the study that deep integration of Web 2.0 services and capabilities into music education will increase objective academic results, motivation and student involvement has been fully confirmed. It has also been revealed that it helps students reduce academic anxiety. The research results can be used as a basis for further research and in the course of the development of music education curricula based on online learning, mobile learning and the integration of music learning into a digital environment.
\end{abstract}

Keywords-Web 2.0, music education, academic anxiety, digitalization 


\section{Introduction}

A change in the structure of music education is taking place all over the world due to the total penetration of digital technologies in the methods of recording and distributing musical content, as well as in the methods of creating musical works [1-4] The advent of Web 2.0 has blurred the very notion of musical professionalism, and the dissemination of content in new ways - through social networks - has changed the concept of monetizing knowledge and skills in this area [5,6]. The structure of the dissemination of musical knowledge has also been transformed, which is closely related to the availability of musical instruments and the ability to play them. Previously, this required a number of special conditions: music instrument maintenance, facilities to practise and play the instrument (special rooms, soundproofing and other requirements), special contact with the teacher taking into account their limited time and the need to organize a meeting, etc. The emergence of digital means of communication, the improvement of bandwidth and the quality of music and video encoding, and the dissemination of educational materials have allowed students to obtain quality music education outside of specialized music educational institutions [7,8]. The development of the musical instrument emulation industry and the improvement of the sound quality of digital emulation (MIDI technology), as well as the emergence of a wide range of non-acoustic technogenic methods of creating music (electronic synthesis, techno, etc.) are of critical importance in this process [9].

According to most researchers, achieving mastery that allows the use of musical skills professionally still requires mentoring and constant contact with other professional musicians. In most cases, there is a need to create groups to develop and promote musical content; thus, the communication factor in music education comes to the fore [10].

The issue of the impact of Web 2.0 on the quality of music education and the psychological state of students has not been fully elaborated despite the fact that online learning and mobile learning in many other professional fields have been carefully studied for many years $[11,12]$. The need to study these factors in music pedagogy is crucial for two reasons. The first reason is the need of a musician to master the Web 2.0 environment as a means of networking, skill development, promotion of their art and even co-creation on the basis of available web services $[1,8,13]$. The second one is the need to master the skills and technologies of digital music: tools for creating, recording, and modifying music, etc. At the very least, any person engaged in the musical culture professionally must be able to competently use these tools [14-16].

\section{Literature Review}

The impact of Web 2.0 on music education has been studied from two perspectives. First of all, digital transformation in the music industry is being studied [4]. Secondly, many researchers are considering the use of certain technologies or methodological approaches related to digitalization or the use of social media in the training of musicians or in their professional activity [12]. 
Digitalization is studied as a phenomenon that has two interpretations. Digitization is the transformation of content, in particular, music and video content into digital format, which has its own characteristics. This content is gaining wider popularity and is changing the creativity monetization scheme. The content can be freely modified and distributed, which, in fact, cannot be controlled [2,17]. Digitalization is also defined as the formation of a culture initially based on digital technologies. This applies, in particular, to the creation of music based on electronic emulation of real instruments or the synthesis of computer-generated sound ensembles that make up a piece of music [2,13]. Digitalization also includes new methods of creating music based on recorded fragments (sampling) or simultaneous creation, broadcasting and combination of musical compositions to create digital works, etc. [11,18].

Researchers often associate the use of Web 2.0 mechanisms with the expanded access to the means of storing, distributing and modifying musical content. In this regard, projects focused on music pedagogy are of particular interest, for example, the creation of open data sources with built-in mechanisms for the inclusion and structuring of educational content aimed at academic learning and self-learning [19].

An important aspect of learning based on Web 2.0 and integrated cloud services is a significant increase in the number of tools used in training. These refer to both emulators of musical instruments that simplify the training process, and various ways to present information: video, audio, text, and mixed formats [9,20]. Learning often involves network collaboration in the educational process [1,19]. Research has already demonstrated the great potential of the methods of co-creating music or performing music simultaneously through web services. It should be noted that an adequate methodology for using these new learning opportunities and their integration into music education is being developed now [6,21].

The direct impact of social media on academic performance in music disciplines has not been properly studied, and this gap is expected to be partially eliminated in the present research. The research novelty and contribution are related to the study of the impact of the integration of Web 2.0 services and the opportunities that social networks provide to musicians during their studies on the academic performance and psychological state.

\section{Methods and Materials}

\subsection{Participants}

The research was conducted at R. Glier Kyiv Municipal Academy of Music (Kyiv, Ukraine). There were two experimental groups (hereinafter Group 1 and Group 2) and a control group. Each group consisted of 50 people with an equal ratio of men and women (50\% each). The sample is representative for the educational institution and provides reliable data - the statistical does not exceed $p=2.64$. The study was conducted during one semester of the 2019-2020 academic year. The participants belonged to 8 different specialties and studied at 8 different departments of the educational institution, which guaranteed the fullest coverage of different types of education 
and curriculum content and allowed us to more accurately assess the impact of Web 2.0 on the academic success of students of various music specialties.

\subsection{Research design}

The research hypothesis is based on the following two assumptions:1) the integration of music education with the services and capabilities provided by Web 2.0 will significantly improve academic achievements of students; 2) it will also increase the quality of involvement and motivation to improve professionalism and knowledge.

Group 1 was placed in the learning environment partially integrated with Web 2.0 services. This implied the creation of a virtual community on a selected social network (Facebook) and a group to communicate with the teachers of specialized subjects; provision of access to shared service accounts and the ability to publish music and video content (YouTube, TikTok, Instagram, etc.); provision of access to the repository of educational content and the work of colleagues through social networks.

Group 2 was trained based on the same program but it had deeper integration with Web 2.0 services. The integration included the services of Group 1 and additionally a structured schedule of online consultations with a teacher; scheduled private consultations with each teacher to discuss issues related to learning; access to online music production services; easy access to joint discussions of educational material, tasks, products of creativity or performance through social networking services; the possibility of getting a preliminary assessment.

The results of the integration of education with Web 2.0 services were evaluated with the help of a pre-test, which was carried out before the start of the experimental program and a post-test, which was carried out within a week after its completion. The test was a standard assessment by a group of qualified teachers of specialized subjects in the given specialty. The assessment was given according to the 12-point scale accepted at the university.

At the end of the experimental training period, there was a survey aimed at the subjective assessment of some important psychological parameters of the participants. They were asked to rate the quality of their involvement in learning musical subjects, the level of motivation to continue learning and improve skills, and the level of academic anxiety on a 5-point scale. These parameters were estimated based on the results of the experiment and there are traces of the impact of partial or deeper integration of the traditional training program with the services and capabilities of Web 2.0.

Only the participants of experimental groups were surveyed to obtain their subjective assessment of the usefulness of introducing Web 2.0 into the educational process. They were asked to rate 5 questions on the classic psychometric 5-point Likert scale: "Web 2.0 is useful for my music education"; "Web 2.0 contributes to my musical development and learning"; "Web 2.0 develops personal relationships and connections"; "I need more Web 2.0 services in the training program"; "Web 2.0 fosters a relationship with the teacher." The grades were evaluated on a scale from 0 ("completely disagree") to 5 points ("absolutely agree"). The average value of the responses obtained was separately evaluated for each group. 
Due to the need to clarify some of the results obtained, we conducted an additional unrepresentative and unstructured survey of some participants, which can become the basis for further studies and the results of which are also included in this study.

\subsection{Ethical issues}

All participants were selected from among those who agreed to volunteer. The conditions and objectives of the study were explained to the participants and the anonymity of participation was guaranteed. No personal data were used, collected, or saved during the experiment. Only aggregate data collected based on learning outcomes were evaluated; they were not associated with the personalities of the participants. Participation in the study did not affect the assessment or the quality of knowledge obtained in terms of the quality of mastering the standard curriculum.

\subsection{Instruments}

The statistical analysis of the data obtained during the study, as well as their visualization were carried out in Microsoft Excel 2013.

\subsection{Research limitation}

Although the study is representative, it involves only one university and therefore its results should be extrapolated to a wider sample with appropriate limitations. In addition, the study did not differentiate participants by social groups, in particular, according to gender, origin, social status, and other parameters that could significantly clarify the results obtained.

\section{$4 \quad$ Results}

The results of the objective knowledge test are of particular interest in this context as the participants were assessed according to the traditional system after evaluating their musical achievements, theoretical knowledge and abilities. Thus, the uniformity of assessment by teachers and the three groups of participants was ensured. Significant progress of the experimental groups compared to the control one can be observed in Fig. 1. The root-mean-square deviation in the results of pre-test and post-test in Group 1 did not exceed 0.82, in Group 2 - 0.91; thus, higher post-test scores are statistically significant and not random. It can be confidently stated that the groups that used the services and capabilities of Web 2.0 demonstrated better results. The difference in the average post-test results of Group 1 and Group 2 also falls within the statistical error and standard deviation of the results of both groups. Thus, the higher results obtained by Group 2, which was trained based on deeper and more structured integration of learning with Web 2.0 services, are also statistically significant. According to these results, the first part of the research hypothesis has been confirmed. 


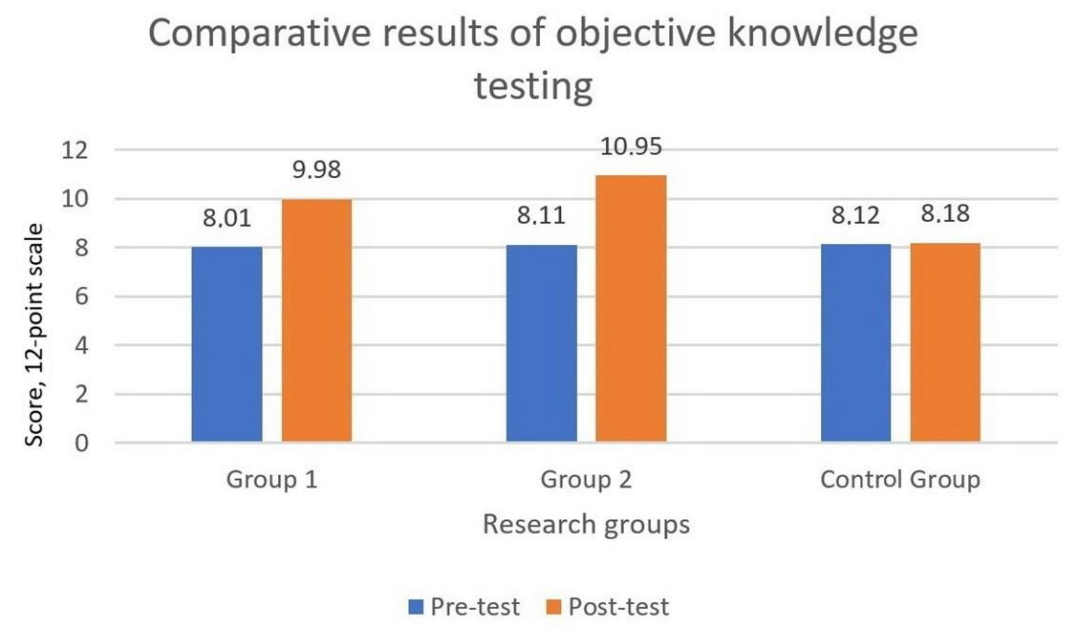

Fig. 1. Comparative results of objective knowledge testing of all participant groups.

The result of the study of some of the most important psychological parameters in the learning process also demonstrates a significant psychological support of Web 2.0 for the academic achievements of emerging musicians. It should be noted that the average level of all the studied parameters from the perspective of the students' subjective assessment of their condition turned out to be quite high. Even in the control group, the level of motivation and involvement significantly exceeded the average mark (3.79 and 3.54, respectively). However, the average level of academic anxiety in the control group was significantly higher compared to the two experimental groups and amounted to 3.98 - the highest indicator in the three groups. The highest level of anxiety in the group where learning was not integrated with Web 2.0 is statistically significant: in the three groups, the standard deviation of the indicator does not exceed 0.54 , which indicates the impossibility of a statistical error in this test.

The level of subjective assessment of motivation and involvement in the three groups is within the possible statistical error with due regard to the value of the standard deviation for each test - it does not exceed 0.61 in the study of involvement and 0.65 in the study of motivation (Fig. 2). Only the difference between the assessments of Group 2 and the control group does not fall within the error. Based on this, we can confidently state that the deep integration of music education with Web 2.0 leads to an increase in students' motivation and involvement in the development of their specialty while the introduction of less deep integration may not ensure such a result. 


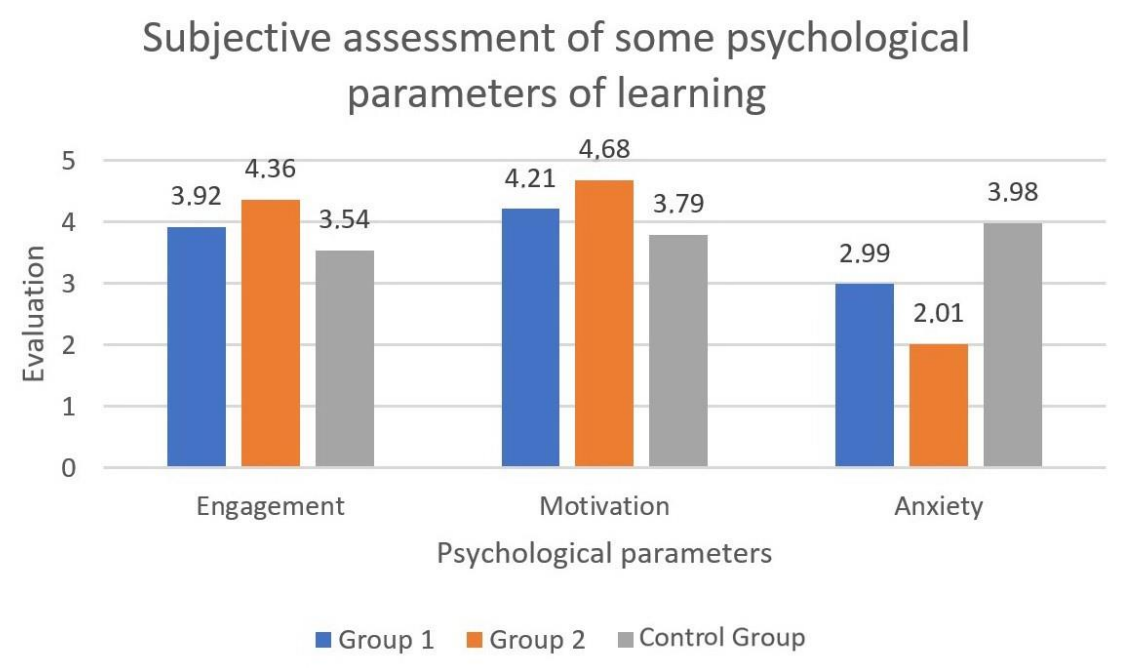

Fig. 2. Subjective assessment of some psychological parameters of learning.

The subjective assessment of the impact of the integration of music education with Web 2.0 demonstrates students' confidence in positive results (Fig. 3). Undoubtedly, it should be borne in mind that this is the subjective opinion of the respondents which is supported by the results of objective knowledge testing (Fig. 1). Considering the mean deviation for each questionnaire question, it should be noted that all of them are statistically significant as the deviation for all questions does not exceed 0.69 , which is much less than the difference between the assessments of Group 1 and Group 2 in the survey. Thus, we can conclude that Group 2, which received deeper Web 2.0integrated training, more confidently assesses the positive results of the integration. In addition, the assessment of the impact of Web 2.0 on learning by both groups was significantly above the average statistical score on the Likert scale - more than 3.0 points were given to each parameter. 


\section{Subjective assessment by students of the quality} of interaction with Web 2.0.

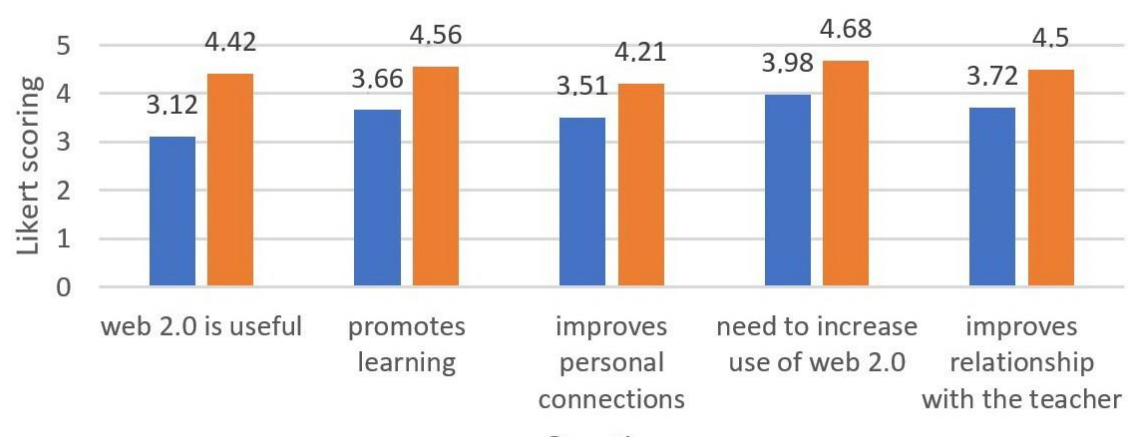

-Group 1 Group 2

Fig. 3. Students' subjective assessment the quality of interaction with Web 2.0.

Based on the research results, it can be stated that both parts of the research hypothesis have been completely confirmed.

The results related to the reduction of academic anxiety were of particular interest as they were not expected and included in the research hypothesis. The participants were asked to fill in an unstructured questionnaire and state their own views on the reasons for reduced anxiety. The assessments of the participants can be summarized as follows:

1. Closer communication on social networks with a focus on vocational training improves awareness and eliminates fear of the unknown;

2. Students receive more personal support from fellow students and friends from other courses and areas of study;

3. More frequent communication with the teacher and their encouragement and assessment contributes to psychological comfort of learning;

4. Students receive more objective information about difficulties and complex topics, as well as more tools to overcome them.

Thus, we can say that the integration of social networks into education provides many additional tools to improve the quality of education.

Further research can be focused on a more accurate differentiation of the influence of certain Web 2.0 forms and technologies on music education and the use of these elements in educational programs. It is also necessary to expand the study of the features of the impact of the Web 2.0 integration into the curriculum from the perspective of different social groups based on gender, socio-economic status of students, vocational guidance and specialization, etc. This will make music education more effective and allow students to actively integrate into the digital environment and modern digital culture and economy while still being involved in the learning process. 


\section{Discussion}

The results of the study correlate with the more limited results of the application of certain pedagogical techniques and technologies associated with digitalization or Web 2.0 in the field of music pedagogy.

The development of three-dimensional representation of information and the improvement of its transmission channels led to the fact that visual representation was proposed to be used in music education, and this approach demonstrated good results [22]. Visual representation is widely used on social media, in particular on music YouTube channels, as a means of visualizing the process of playing a particular musical instrument or visualizing the musical and melodic structure while playing a melody [23-25].

Specialized web applications for teaching music analysis and composition are especially effective [26]. Such specialized services and programs often provide an opportunity to simplify learning, for example by offering automation of the accompaniment, which is better and more satisfying compared to a live partner musician [23,24]. We did not conduct a separate analysis by specialty, but this can be considered the most important task for further research as current studies have proven the qualitative result of the use of these applications $[3,7,23,24]$. Their specialized and often paid nature can be considered a certain limitation while in our study we used only free applications, which also ensured the expected positive result.

A number of researchers note the importance of using web technologies for teaching and promoting musical culture, which they call "musical cultivation". This approach is adequate given the significant role of music in the life of several social strata, including adolescents, young women, and a number of social groups of middleaged professionals $[27,28]$. The use of web content and distribution mechanisms to create a higher and more complex musical culture requires training and accustoming large audiences to the best examples of music culture. This is facilitated by the high interest of people who constantly use social networks in music education -at least in its basics $[9,20]$.

All researchers devote their studies to different forms of blended music learning. It is almost impossible to find research related exclusively to online or mobile music education at a professional level. Researchers unanimously agree that professional training in this area requires classical classroom learning, face-to-face contact with teachers, mentoring, as well as direct contact with physical tools and equipped classrooms $[15,29]$. Blended learning allows students to significantly improve results and academic performance, and also much earlier integrates them into the professional and creative life. This raises the general level of musical education and increases musical diversity in the general cultural context [27].

The impact of academic cognitive load and the specifics of academic anxiety in the field of music pedagogy has not been properly considered despite the fact that these topics have been carefully studied by researchers for the natural sciences and humanities learning cycle [11,30,31]. In this case, our research cannot be considered a fullfledged study as this area has only partially been covered. However, interesting results have been obtained and statistically confirmed. 


\section{Conclusion}

The integration of Web 2.0 services and capabilities into music education is determined by the total digitalization of all spheres of culture and the penetration of digital technologies not only into the process of recording and distribution, but also into the process of creating musical works. Web 2.0 creates a completely new distributed culture of musical creativity and provides musicians with new opportunities for creativity and distribution of their creative products. The study was devoted to the impact of the integration of mass services and capabilities provided by Web 2.0 on the objective quality of music education, as well as the most important psychological parameters of music university students and their professional development. The study involved 150 students of R. Glier Kyiv Municipal Academy of Music (Kyiv, Ukraine); there were three groups of 50 participants: two experimental groups and a control one. In Experimental Group 1, training was partially integrated with Web 2.0 services; in Experimental Group 2, there was a more complete and structured integration. Based on the results of semester training and the objective test scores, a significant increase in the results of experimental groups was observed (an increase by 1.3 points in Group 1 and 2.84 points in Group 2 on a 5-point scale); Group 2 demonstrated a more significant improvement. The subjective assessment of the level of involvement, motivation and anxiety showed a statistically significant decrease in academic anxiety in the experimental groups (2.99 and 2.01 compared to 3.98 in the control group, respectively) and a statistically significant increase in motivation and involvement in the group with deep integration of Web 2.0 services (Experimental Group 2). The research results can be used as a basis for further research and in the course of the development of music education curricula based on online learning, mobile learning and the integration of music learning into a digital environment.

\section{$7 \quad$ References}

[1] Cremata, R., Powell, B. (2017). Online music collaboration project: Digitally mediated, deterritorialized music education. International Journal of Music Education, 35(2): 302315. https://doi.org/10.1177/0255761415620225

[2] Strachan, R. (2017). Sonic technologies: popular music, digital culture and the creative process. Bloomsbury Publishing USA.

[3] Zhang, P., Sui, X. (2017). Application of digital music technology in music pedagogy. International Journal of Emerging Technologies in Learning, 12(12): 4-13.

[4] Baratè, A., Haus, G., Ludovico, L.A., Pagani, E., Scarabottolo, N. (2019). 5G Technology and Its Applications to Music Education. In Procs 13th International Conference eLearning 2019, pp. 65-72. https://doi.org/10.33965/el2019_201909f009

[5] Boumba, M.S. (2018). Participative Web 2.0 and Second Generation Congolese Youth in Brussels: Social Network Sites, Self-Expression, and Cultural Identity. In Diaspora and Media in Europe. Palgrave Macmillan, Cham, pp. 165-183. https://doi.org/10.1007/978-3319-65448-5_8

[6] Greener, G., Salcum, S., Goldberg, D. (2019). Electronic Word of Mouth and the Music Industry in the Age of Web 2.0. SocArXiv, 11. https://doi.org/10.31235/osf.io/7wqhd 
[7] Gall, M. (2017). Technology in music education in England and across Europe. In the Oxfordhandbook of technology and music education. Oxford University Press, pp. 31-48. https://doi.org/10.1093/oxfordhb/9780199372133.013.2

[8] Horsley, S., Waldron, J. (2017). Challenging music education: The transformative potential of social media. In Proceedings of the 8th International Conference on Social Media \& Society, pp. 1-5. https://doi.org/10.1145/3097286.3097329

[9] Ludovico, L.A. (2017). The Web MIDI API in On-Line Applications for Music Education. In Proceedings of the Ninth International Conference on Mobile, Hybrid, and On-line Learning (eLmL 2017), Luca Andrea Ludovico and Ahmed Mohamed Fahmy Yousef (Eds.). IARIA XPS, pp. 72-77. https://doi.org/10.24247/ijcmsoct20174

[10] Liyakat, M. (2017). Understanding Web 2.0: The Extensions of Emotional Release And Status: Uses And Gratification Theory In The Context Of Kashmir. International Journal of Communication and Media Studies, 7(4): 21-28.

[11] Ruthmann, A., Mantie, R (2017). The Oxford handbook of technology and music education. Oxford University Press. https://doi.org/10.1093/oxfordhb/9780199372133.001.0001

[12] Webster, P.R. (2020). Software to Interrogate Qualitative Data in Music Education. In Collecting, Analyzing and Reporting Data: An Oxford Handbook of Qualitative Research in American Music Education, Volume 2. Oxford University Press, pp. 117-129. https://doi. org/10.1093/oxfordhb/9780199844272.013.017

[13] Burland, K. (2020). Music for all: Identifying, challenging and overcoming barriers. Music \& Science, 3: 2059204320946950. https://doi.org/10.1177/2059204320946950

[14] Avery, A. (2016). Just do it! Web 2.0 and the breaking of the tacit dimension for knowledge acquisition. In SAIS 2016 Proceedings of the Southern Association for Information Systems Conference, Paper (Vol. 1).

[15] Della Ventura, M. (2017). Creating inspiring learning environments by means of digital technologies: A case study of the effectiveness of WhatsApp in music education. In ELearning, E-Education, and Online Training. Springer, Cham, pp. 36-45. https://doi.org/ 10.1007/978-3-319-49625-2_5

[16] Della Ventura, M. (2018). Twitter as a music education tool to enhance the learning process: conversation analysis. In New Media for Educational Change. Springer, Singapore, pp. 81-88. https://doi.org/10.1007/978-981-10-8896-4_7

[17] Li, Z. (2017). Design of Online Vocal Music Course Based on Azure-PaaS Platform. International Journal of Emerging Technologies in Learning, 12(9): 17-27. https://doi.org/ 10.3991/ijet.v12i09.7495

[18] Buzás, Z., Sagrillo, D. (2019). The Role of Cultural Heritage in 21st-Century Music Education. Polgári Szemle: Gazdasági És Társadalmi Folyóirat, 15: 317-328. https://doi. org/10.24307/psz.2020.0218

[19] Caixinha, H., Caixinha, S. (2019). Connecting the worlds of music with a digital repository for collaborative research, education and content dissemination. In Proceedings of Edulearn19 Conference, 1st-3rd July 2019, Palma, Mallorca, Spain, pp. 1837-1847. https://doi.org/10.21125/edulearn.2019.0520

[20] Pauwels, J., Sandler, M.B. (2019). A web-based system for suggesting new practice material to music learners based on chord content. In IUI Workshops'19, March 20, 2019, Los Angeles, USA, pp. 1-4.

[21] Sagrillo, D. (2019). Cultural Heritage, Diversity, Functionality, Education of Music in a European Context. In Music Education in the Focus of Historical Concepts and New Horizons. John Von Neumann University, pp. 106-111.

[22] Kritsis, K., Gkiokas, A., Acosta, C.Á., Lamerand, Q., Piéchaud, R., KaliakatsosPapakostas, M.A., Katsouros, V. (2018). A web-based 3D environment for gestural inter- 
action with virtual music instruments as a STEAM education tool. In NIME, pp. 348-349. https://doi.org/10.1145/3243274.3243297

[23] Li, Y. (2020). Application of Computer-Based Auto Accompaniment in Music Education. International Journal of Emerging Technologies in Learning, 15(6): 140-151.

[24] Li, Y. (2020). Visual Education of Music Course for College Students Based on HumanComputer Interaction. International Journal of Emerging Technologies in Learning, 15(2): 175-186. https://doi.org/10.3991/ijet.v15i02.12535

[25] Yan, B., Zhou, Q. (2017). Music learning based on computer software. International Journal of Emerging Technologies in Learning, 12(12): 142-150.

[26] Barate, A. (2017). A Web-based Petri Nets Application to Teach Music Analysis and Composition. In International Conference on Mobile, Hybrid, and On-line Learning. IARIA XPS Press, pp. 78-83.

[27] Crawford, R. (2017). Rethinking teaching and learning pedagogy for education in the twenty-first century: blended learning in music education. Music Education Research, 19(2): 195-213. https://doi.org/10.1080/14613808.2016.1202223

[28] Cawley, J. (2018). Using Web-Based Technologies during Musical Enculturation: Some Educational Implications for the Learning of Irish Traditional Music. In Proceedings of the 19th International Seminar of the ISME Commission on Music Policy: Culture, Education, and Media Ludwig-Maximilians-Universitaet, Munich, pp. 32-42.

[29] Ruokonen, I., Enbuska, J., Hietanen, L., Tuisku, V., Rimppi, A., Ruismäki, H. (2017). Finnish student teachers' self-assessments of music study in a blended learning environment. The Finnish Journal of Music Education, 20(2): 30-39. https://doi.org/10.15405/ ejsbs. 249

[30] Diaz, F.M. (2018). Relationships among meditation, perfectionism, mindfulness, and performance anxiety among collegiate music students. Journal of Research in Music Education, 66(2) 150-167. https://doi.org/10.1177/0022429418765447

[31] Dobos, B., Piko, B.F., Kenny, D.T. (2019). Music performance anxiety and its relationship with social phobia and dimensions of perfectionism. Research Studies in Music Education, 41(3): 310-326. https://doi.org/10.1177/1321103x18804295

\section{Author}

Revenko Vira Alekseevna is a Professor of the Department of Academic Singing and Choral Conducting, R. Glier Kyiv Municipal Academy of Music, Kyiv, Ukraine

Article submitted 2020-11-06. Resubmitted 2020-12-11. Final acceptance 2020-12-12. Final version published as submitted by the authors. 